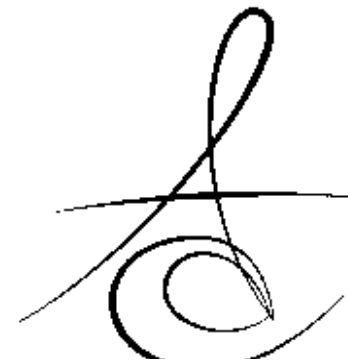

Makale Kodu/Article code: 1035

Makale Gönderilme tarihi; 15.01.2013

Kabul Tarihi: 16.04.2013

\title{
ENDODONTİDE KONİK IŞINLI BİLGİSAYARLI TOMOGRAFİ
}

\section{CONE BEAM COMPUTED TOMOGRAPHY IN ENDODONTICS}

Yrd. Doç.Dr. Elif Tarım ERTAŞ*

Yrd. Doç.Dr. İsmail Davut ÇAPAR*
Yrd. Doç.Dr. Hakan ARSLAN**

Araş. Gör. Dt. Tuba GÖK***

\author{
Yrd. Doç.Dr. Hüseyin ERTAŞ**
}

\section{ÖZET}

Dişin anatomisi ve çevre dokuların değerlendirilmesi için alınan radyografiler endodontik problemlerin çözümünde önemli rol oynamaktadır. Ancak geleneksel radyografi teknikleri sıkıştırılmış alanda 2 boyutlu görüntü imkânı sağlamaktadır. Konik ışınlı bilgisayarlı tomografi (cone beam computed tomography, CBCT) geleneksel bilgisayarlı tomografi cihazlarına göre daha az maliyette üç boyutlu görüntü oluşturabilen cihazlardır. Bu derlemenin amacı endodonti pratiğinde $\mathrm{CBCT}^{\prime}$ nin muhtemel uygulamaları üzerine bilgi sunmaktır.

Anahtar Kelimeler: Konik ışınlı bilgisayarlı tomografi; Endodonti; Kök kanalı

\section{ABSTRACT}

The radiographs, which were taken for assessing the anatomy of the tooth and its surrounding anatomy, play an important role in the solution of endodontic problems. However conventional radiography techniques provide two dimensional compressed images. CBCT generates three dimensional images at lower cost than conventional computed tomography. The aim of this review is to provide information on $\mathrm{CBCT}$ and its potential applications in endodontic practice.

Keywords: Cone Beam Computed Tomography; Endodontics; Root canal

\section{GİRİ̧}

Endodontide radyografik değerlendirme teşhis, tedavi planlanması ve sonuçların değerlendirilmesi için önemli bir aşamadır. Geleneksel radyografiler üç boyutlu anatomiyi iki boyutlu görüntüye sıkıştırmaları ${ }^{1}$, geometrik distorsiyonlara sebep olmaları², anatomik bölge süperpozisyonlarına maruz kalmaları ${ }^{3}$, küçük periapikal lezyonların teşhisinde yetersiz olmaları ${ }^{4}$, alınan radyografilerde standardizasyon sağlayamamaları ${ }^{5}$ ve filmin ağız içerisine uygun bir şekilde yerleştirilmesinin mümkün olmadığı durumlarda yetersiz kalmaları gibi sınırlamalara sahiptirler.

1982 yılında ilk kez anjiyografi amacıyla kullanılan konik ışınlı bilgisayarlı tomografi (cone beam computed tomography, CBCT) maxillo-fasiyal bölgenin görüntülenmesi amacıyla ilk kez 1997 yılında kullanılmıştır. $^{6}$
CBCT çene yüz sisteminin üç boyutlu taranabilmesine imkân sağlayan ekstra-oral görüntüleme tekniğidir. Her ne kadar CBCT geleneksel bilgisayarlı tomografi (computed tomography, CT)'den orijin almış olsa da, geleneksel CT cihazlarına göre CBCT'nin kullanımı daha basit olup daha ucuz bir donanıma sahiptir. En önemli avantajı alınan radyasyonun önemli derecede azaltılmasıdır. Bu durum tarama süresinin daha kısa olması, ışınlama olayının çok hızı olması ve görüntü sensörlerinin oldukça gelişmiş olmasından kaynaklanmaktadır ${ }^{7}$. Ayrıca geleneksel CT cihazları helezonik ışın kullanılmaktadır ancak CBCT cihazında hastanın çevresinde $360^{\circ}$ rotasyon elde etmek amacıyla konik ışınlı radyasyon kullanılmaktadır.

CBCT cihazları görüntü alanına (field of view, FOV) veya tarama sırasındaki hasta pozisyonuna göre sınıflandırılmaktadır. FOV sınıflamasına göre; geniş

\footnotetext{
* İzmir Katip Çelebi Üniversitesi, Diş Hekimliği Fakültesi, Ağız Diş ve Çene Radyolojisi Anabilim Dalı, İzmir, Türkiye

** İzmir Katip Çelebi Üniversitesi, Diş Hekimliği Fakültesi, Endodonti Anabilim Dalı, İzmir, Türkiye.

*** İzmir Katip Çelebi Üniversitesi, Diş Hekimliği Fakültesi, Endodonti Anabilim Dalı, İzmir, Türkiye.
} 
FOV $(>15 \mathrm{~cm})$, orta FOV $(10-15 \mathrm{~cm})$ ve küçük FOV $(<5 \mathrm{~cm})$ olmak üzere üç gruba ayrılmaktadır. Endodontik kullanıma daha uygun olduğu belirtilen küçük FOV cihazlar günümüzde giderek artan bir popülariteye sahiptirler. Tarama sırasındaki hasta pozisyonuna göre ise; sırtüstü, oturarak ve ayakta olmak üzere üç sınifta toplanmaktadır. ${ }^{8}$

CBCT cihazlarında uygulanan etkili radyasyon dozu cihazın tipine, teknik ayarlara (mAs, kVp), ışının geometrisine-tipine (aralıklı, devamlı vs.) ve görüntünün alındığı bölgeye göre değişebilmektedir. Bu değer yapılan geçmiş çalışmalarda 5,3-860 $\mu \mathrm{Sv}$ arasında değişmekte ve yaklaşık olarak 0,3-61 dijital panoramik filme eşdeğer olabilmektedir. ${ }^{9-11}$

CBCT'nin endodontik tedavide kullanımıyla ilgili literatürde birçok çalş̧ma ve ilginç sonuçlar bulunmaktadır. Bu derlemenin amac $\mathrm{CBCT}^{\prime}$ nin günümüze kadar endodonti pratiğinde muhtemel kullanım alanlarının açıklanmasıdır.

\section{Endodontide CBCT Kullanımı Apikal periodontitisin tespiti}

Periapikal lezyonların teşhisi, lokalizasyonu ve boyutlarının değerlendirilmesi endodontik tedavi planı ve kontrol radyografilerinin doğru bir şekilde yorumlanması bakımından önemlidir. ${ }^{12}$ Ancak kök kanal eneksiyonu sonrasında ortaya çıkan periapikal lezyonların tümü özellikle erken dönemde radyografik olarak tespit edilememektedir. ${ }^{13,14}$

Periapikal lezyonların tespitinde CBCT geleneksel radyografilerden daha hassastır. Patrick ve ark. ${ }^{15}$ CBCT cihazlarının 0,8 mm'den büyük lezyonların tespitinde geleneksel radyografilerden daha iyi sonuçlar verdiğini bildirmişlerdir. 0.8 mm'den küçük lezyonlarda geleneksel radyografiler gibi CBCT cihazlarının da limitasyonlara sahip olduğunu ifade edilmiştir. Estrela ve ark. ${ }^{13}$ periapikal lezyon teşhisinde CBCT cihazlarının etkinliğinin panoramik ve periapikal radyografilerden daha iyi olduğunu belirtmişleridir.

CBCT metodunda periapikal kemik lezyonunun iyileşmesi daha önceden tespit edilen iyileşmelerden daha uzun sürebilmektedir. Christiansen ve ark. ${ }^{16}$ periapikal kemik yıkılımın tedavi sonrası iyileşmesini geleneksel periapikal radyografi ve CBCT kullanarak değerlendirmişlerdir. Buna göre yapılan tedaviden 1 yıl sonraki kontrolde periapikal radyografilerle iyileşme görülürken CBCT ile hala tam iyileşme görülmemektedir.

\section{Endodontik cerrahi öncesi değerlendirme}

CBCT diğer cerrahi işlemlerde olduğu gibi endodontik cerrahi işlemler öncesi kök uzunluğu-açısal şekli, kortikal kemik kalınlığı, maksiller sinüs, mandibular kanal ve mental foramen gibi komşu anatomik yapıların doğru bir şekilde incelenmesinde çok yararlı bir araçtır. ${ }^{17,18}$

\section{Dental travmaların değerlendirilmesi}

Dental travmaların doğru bir şekilde teşhisi tedavinin prognozu açısından önemlidir. Dental travmalar sonucunda genellikle birden fazla diş etkilenmektedir. Herhangi bir kırı̆ı̆ gözlemlenebilmesi için farklı açıdan birden fazla periapikal film çekilmesi gerekmektedir. Buna rağmen periapikal radyografiler ile bu kırığın tespit edilememe intimali mevcuttur. Yeni travma geçirmiş olan hastalarda ekstraoral bir teknik olan CBCT metodu ile görüntü alınması ağız içinden birden fazla periapikal radyografi alınmasından daha kolaydır. Ayrıca horizontal kök kırıklarının tespitinde CBCT'nin periapikal radyografilerden daha hassas olduğu bildirilmiştir. ${ }^{19}$ Vertikal kök kırıklarının (VKK) tespitinde endodonti pratiğinde genellikle güçlük yaşanmaktadır. Bazen kök kırı̆ı mevcut olduğu durumlarda klinik ve radyografik bulgular kök kırı̆ı tespit edilene kadar gizli kalabilmektedir. VKK'nın uzun zamandır mevcut olması durumunda dahi klinik olarak herhangi bir bulgu görülemeyebilir. Bölgesel olarak izole olmuş derin ve ince bir periodontal cep durumunda VKK düşünülebilir. ${ }^{20}$ Bununla birlikte periodontal sondun defekt üzerine yerleştirilmesi güç olduğundan VKK'nın tespiti gözden kaçabilir. VKK vakalarında görülen J ve hale şeklindeki radyolüsent alanlar kemikte lezyonun görünmesi içi gerekli miktarda yıkım gerçekleşmeden tespit edilemeyebilir. Ayrıca apikal periodontitisli vakalarda benzer şekillerde görülen radyolüsent alanlar VKK ile karıştırılabilir ${ }^{21}$. Travma sonucunda meydana gelen kök kırıklarının tespit edilememesi bu dişlerin uygun olmayan bir şekilde tedavi edilmesi ile sonuçlanacaktır. Yapılan ex-vivo çalışmalarda VKK'nın CBCT ile periapikal radyografilerden daha hassas tespit edildiği gösterilmiştir. ${ }^{22}$

CBCT dento-alveolar travmaların teşhisinde faydalı olabilmektedir. ${ }^{1,23,24}$ Alveolar lüksasyon yaralanmalarının yapısı ve şiddeti CBCT metodu ile herhangi bir geometrik distorsiyonuna ve anatomik görüntü bozukluğuna uğramadan tespit edilebilmektedir. 
Lüksasyon yaralanmalarına bağlı olarak meydana gelen yer değiştirmelerin derecesi ve yönü CBCT yöntemi ile kolaylıkla tespit edilebilir.

Ayrıca Cohenca ve ark. ${ }^{25}$ dental travma geçirmiş olan üç hasta üzerinde $\mathrm{CBCT}$ metodu ile görüntü almışlar, klinik ve radyografik muayene ile tespit edilemeyen kortikal kemik kırıklarını tespit edebilmişlerdir.

\section{Kök kanal anatomisinin değerlendirilmesi}

\section{C-Şekilli Kanalların Tespitinde}

Özellikle alt ikinci molar dişlerde sıklıkla görülen C-şekilli kanal, kök füzyonu sonucu ortaya çıkan anaomik bir varyasyondur. Kök kanal sisteminin yerinin lokalize edilmesi, temizlenmesi ve şekillendirilmesinde zorluklar yaşanan bu tip kanallarda preoperatif CBCT uygulamasının endodontik tedaviyi kolaylaştırabildiği belirtilmiştir. $^{26}$

2. Aksesuar Kök Kanalları ve Fazla-Eğri Kökler Klinik veya radyografik olarak tespit edilemeyen ekstra kök kanalları veya aşırı eğri kökler endodontik tedavide başarısızlıklara sebep olabilmektedir ${ }^{27}$. Bu tip durumlarda da CBCT iyi sonuçlar vermektedir. ${ }^{26,28}$

\section{Diş anatomisindeki farklılıkların teşhisi}

1. Dens Invaginatus (DI)

DI mineralizasyon fazı öncesinde mine organının dental papilla içine doğru kıvrılması sonucu meydana gelen gelişimsel bir anomalidir. Özellikle köke uzanan tip III DI klinik olarak öneme sahiptir. Keles ve Cakıcı ${ }^{29}$ periapikal lezyona ve DI'a sahip üst lateral dişin sadece DI kısmının tedavi edilip pulpanın canlı korunabildiğini göstermişlerdir. Bu nedenle doğru teşhis ve tedavi bakımından bu tür vakalarda CBCT kullanımı önerilmektedir. ${ }^{30}$

\section{Palatoginvival Oluk:}

Palatogingival oluk; maksillar santral ve lateral kesici dişlerin periodontal cep ve kemik yıkımı ve bunlarla birlikte pulpa nekrozunun görülebildiği nadir bir gelişimsel anomalisidir ${ }^{31}$. Mine, dentin veya sement tabakasının ince olmasına bağlı olarak pulpa dokusu irritanlardan etkilenerek nekroze olabilir 32. Periapikal radyografiler iki boyutlu görüntü verdikleri için bu gibi gelişimsel anomalilerin teşhisinde yetersiz kalmaktadırlar. Üç boyutlu görüntülemeye olanak sağlayan CBCT'nin gelişmesi ile bu tür vakaların erken teşhisi ve tedavisi mümkün olmuştur. ${ }^{33}$

\section{Komplikasyonların Teşhisi}

\section{Parestezi}

Lokal anestezi, aşırı instrumentasyon veya taşkın dolgu gibi durumlar paresteziye neden olabilmektedir. ${ }^{34}$ Bunlardan taşkın dolgudan kaynaklanan parestezilerde taşkın dolgu geleneksel radyografilerle tespit edilmeyebilmektedir. Bu gibi durumlarda CBCT'nin etkili bir teşhis cihazı olduğu belirtilmiştir. ${ }^{35}$

\section{Sodyum Hipoklorit Kazası}

Behrent ve ark. ${ }^{36}$ bir vakada kanal tedavisi boyunca sodyum hipokloritin aşırı instrumentasyon nedeniyle apeksten taştığını ve $\mathrm{CBCT}$ görüntüsünde radyolüsent kabarcıklar şeklinde gözüktüğünü ifade etmişlerdir.

\section{Rezorbsiyonlar}

\section{Eksternal Rezorbsiyon}

Eksternal rezorbsiyon dental dokuların fizyolojik veya patolojik rezorbsiyonu sonucu oluşur. Geleneksel radyografilerle kök rezorbsiyonların yaklaşık \% 65'i yanlış teşhis edilmektedir. Estevez ve ark. ${ }^{37}$ CBTC cihazlarının eksternal rezorbsiyonun teşhisinde geleneksel radyografilere göre daha üstün sonuçlar verdiğini bildirmişlerdir.

\section{Internal rezorbsiyon}

Pulpal enflamasyon sonucunda internal rezobsiyon meydana gelmektedir. Bhuva ve ark. ${ }^{38}$ klasik radyografi ile tespit edilemeyen perforasyona sahip internal rezorbsiyonun teşhis ve tedavisinde $\mathrm{CBCT}$ nin faydalı olduğunu göstermiştir.

\section{Diğer}

\section{Odontojenik Maksiller Sinüzit}

Maksiller sinüzit enfeksiyonları; maksiller diş enfeksiyonları, çekimler, yabancı cisimler veya dental materyallerden kaynaklanabilmektedir. Cymerman ve ark. $^{39}$ CBCT'nin odontojenik maksiller sinüzit vakalarının teşhis edilmesinde faydalı olduğunu ifade etmişlerdir.

Bunların dışında CBCT'nin atipik fasiyal ağrının, non odontojenik lezyonların ayırıcı tanısında, kırık alet, taşkın kanal dolgusu, perforasyonlar, kalsifiye kanal tespiti gibi durumlarda faydalı olduğu bildirilmiştir. ${ }^{40,41}$

\section{CBCT'nin Limitasyonları}

CBCT yönteminin dezavantajı yüksek maliyetli olması ve periapikal grafilere kıyasla daha yüksek radyasyona sahip olmasıdır. 
CBCT teşhis açısından klinik olarak yardımcı olmakla birlikte özellikle de kanal içi metalik postlar kullanıldığı zaman bazı limitasyonlar bildirilmiştir. ${ }^{42} \mathrm{Me}-$ talik post etrafında oluşan artefaktlar incelenen kökteki perforasyonların ve lezyonların teşhisini güçleştirmektedir. ${ }^{43}$

\section{SONUÇ}

Sonuç olarak CBCT endodontik problemlerin teşhis ve tedavisinde yararlı bir araçtır. Bu üç boyutlu görüntüleme tekniği geleneksel radyografi tekniklerinin limitasyonlarının üstesinden gelmekte ve klinisyene ek yararlar sağlamaktadır. Ancak CBCT uygulamalarındaki etkili radyasyon dozunun geleneksel radyografi tekniklerinde kullanılan dozdan daha yüksek olduğu unutulmamalıdır. Bu nedenle CBCT rutin olarak her hastada uygulanmamalı, endodontik tedavi süresince CBCT kullanımı her bir vakada dikkatli bir şekilde analiz edilerek klinik teşhis araçları ve geleneksel radyografi ile yeterli bilgi elde edilemediği zaman tercih edilmelidir.

\section{KAYNAKLAR}

1. Cohenca N, Simon JH, Roges R, Morag Y, Malfaz JM. Clinical indications for digital imaging in dentoalveolar trauma. Part 1: traumatic injuries. Dent Traumatol 2007;23:95-104.

2. Forsberg J, Halse A. Radiographic simulation of a periapical lesion comparing the paralleling and the bisecting-angle techniques. Int Endod J 1994;27:133-8.

3. Tyndall $\mathrm{DA}$, Clifton $\mathrm{TL}$, Webber $\mathrm{RL}$, Ludlow JB, Horton RA. TACT imaging of primary caries. Oral Surg Oral Med Oral Pathol Oral Radiol Endod 1997;84:214-25.

4. Schwartz SF, Foster JK, Jr. Roentgenographic interpretation of experimentally produced bony lesions. I. Oral Surg Oral Med Oral Pathol 1971;32:606-12.

5. Lee SJ, Messer HH. Radiographic Appearance of Artificially Prepared Periapical Lesions Confined to Cancellous Bone. Int Endod J 1986;19:64-72.

6. Robb RA. The Dynamic Spatial Reconstructor: An $X$-Ray Video-Fluoroscopic CT Scanner for Dynamic Volume Imaging of Moving Organs. IEEE Trans Med Imaging 1982;1:22-33.
7. Patel S. New dimensions in endodontic imaging: Part 2. Cone beam computed tomography. Int Endod J 2009;42:463-75.

8. Scarfe WC, Levin MD, Gane D, Farman AG. Use of cone beam computed tomography in endodontics. Int J Dent 2009;1-20.

9. Ludlow JB, Ivanovic M. Comparative dosimetry of dental CBCT devices and 64-slice CT for oral and maxillofacial radiology. Oral Surg Oral Med Oral Pathol Oral Radiol Endod 2008;106:106-14.

10. Pauwels R, Beinsberger J, Collaert B, Theodorakou C, Rogers J, Walker A, et al. Effective dose range for dental cone beam computed tomography scanners. Eur J Radiol 2012;81:267-71.

11. Hirsch E, Wolf $U$, Heinicke F, Silva MA. Dosimetry of the cone beam computed tomography Veraviewepocs 3D compared with the 3D Accuitomo in different fields of view. Dentomaxillofac Radiol 2008;37:268-73.

12. Sogur E, Baksi BG, Grondahl HG, Sen BH. Pixel intensity and fractal dimension of periapical lesions visually indiscernible in radiographs. J Endod 2013;39:16-9.

13. Estrela C, Bueno MR, Leles CR, Azevedo B, Azevedo JR. Accuracy of cone beam computed tomography and panoramic and periapical radiography for detection of apical periodontitis. J Endod 2008;34:273-9.

14. Arslan H, Topcuoglu HS, Barutcigil C, Karatas E, Aladag $H$, Koseoglu $M$, et al. Non-surgical endodontic treatments of teeth associated with large periapical lesions Atatürk Üniv Diş Hek Fak Derg 2012;22:61-5.

15. Tsai P, Torabinejad M, Rice D, Azevedo B. Accuracy of cone-beam computed tomography and periapical radiography in detecting small periapical lesions. J Endod 2012;38:965-70.

16. Christiansen R, Kirkevang LL, Gotfredsen E, Wenzel A. Periapical radiography and cone beam computed tomography for assessment of the periapical bone defect 1 week and 12 months after root-end resection. Dentomaxillofac Radiol 2009;38:531-6.

17. Rigolone M, Pasqualini D, Bianchi L, Berutti $E$, Bianchi SD. Vestibular surgical access to the palatine root of the superior first molar: "low-dose cone-beam" CT analysis of the pathway and its anatomic variations. J Endod 2003;29:773-5. 
18. Tsurumachi T, Honda K. A new cone beam computerized tomography system for use in endodontic surgery. Int Endod J 2007;40:224-32.

19. Kamburoglu K, Barenboim SF, Ariturk T, Kaffe I. Quantitative measurements obtained by microcomputed tomography and confocal laser scanning microscopy. Dentomaxillofac Radiol 2008;37:38591.

20. Tamse A, Kaffe I, Lustig J, Ganor Y, Fuss Z. Radiographic features of vertically fractured endodontically treated mesial roots of mandibular molars. Oral Surg Oral Med Oral Pathol Oral Radiol Endod 2006;101:797-802.

21. Durack C, Patel S. Cone beam computed tomography in endodontics. Braz Dent J 2012;23:179-91.

22. Hassan B, Metska ME, Ozok AR, van der Stelt $P$, Wesselink PR. Detection of vertical root fractures in endodontically treated teeth by a cone beam computed tomography scan. J Endod 2009; 35: 719-22.

23. Cotton TP, Geisler TM, Holden DT, Schwartz SA, Schindler WG. Endodontic applications of conebeam volumetric tomography. J Endod 2007; 33: 1121-32.

24. Patel S, Dawood A. The use of cone beam computed tomography in the management of external cervical resorption lesions. Int Endod J 2007; 40:730-7.

25. Cohenca N, Simon JH, Roges R, Morag Y, Malfaz JM. Clinical indications for digital imaging in dentoalveolar trauma. Part 1: traumatic injuries. Dent Traumatol 2007;23:95-104.

26. Kottoor J, Velmurugan N, Ballal S, Roy A. Fourrooted maxillary first molar having C-shaped palatal root canal morphology evaluated using cone-beam computerized tomography: a case report. Oral Surg Oral Med Oral Pathol Oral Radiol Endod 2011;111:41-5.

27. Ayranci LB, Arslan H, Topcuoglu HS. Maxillary first Molar with three canal orifices in MesioBuccal root. J Conserv Dent 2011;14:436-7.

28. Abella F, Mercade M, Duran-Sindreu F, Roig M. Managing severe curvature of radix entomolaris: three-dimensional analysis with cone beam computed tomography. Int Endod J 2011;44:876-85.
29. Keles A, Cakici F. Endodontic treatment of a maxillary lateral incisor with vital pulp, periradicular lesion and type III dens invaginatus: a case report. Int Endod J 2010;43:608-14.

30. Kfir A, Telishevsky-Strauss Y, Leitner A, Metzger Z. The diagnosis and conservative treatment of a complex type 3 dens invaginatus using cone beam computed tomography (CBCT) and 3D plastic models. Int Endod J 2013;46:275-88.

31. Everett FG, Kramer GM. The disto-lingual groove in the maxillary lateral incisor; a periodontal hazard. J Periodontol 1972;43:352-61.

32. Ballal NV, Jothi V, Bhat KS, Bhat KM. Salvaging a tooth with a deep palatogingival groove: an endoperio treatment--a case report. Int Endod J 2007;40:808-17.

33. Raiput A, Talwar S, Chaudhary S, Khetatpal A. Successful management of pulpo-periodontal lesion in maxillary lateral incisor with palatogingival groove using CBCT scan. Indian J Dent Res 2012;23:415-8.

34. Buyukkurt MC, Arslan H, Topcuoglu HS, Omezli MM. Prognosis of a case with paresthesia associated with prolonged touching of an endodontic paste to the inferior alveolar nerve. J Clin Exp Dent 2011;3:377-81.

35. Gambarini G, Plotino G, Grande NM, Testarelli L, Prencipe $M$, Messineo $D$, et al. Differential diagnosis of endodontic-related inferior alveolar nerve paraesthesia with cone beam computed tomography: a case report. Int Endod J 2011;44:176-81.

36. Behrents KT, Speer ML, Noujeim M. Sodium hypochlorite accident with evaluation by cone beam computed tomography. Int Endod J 2012;45:492-8.

37. Estevez R, Aranguren J, Escorial A, de Gregorio C, De La Torre $F$, Vera J, et al. Invasive cervical resorption Class III in a maxillary central incisor: diagnosis and follow-up by means of cone-beam computed tomography. J Endod 2010;36:2012-4.

38. Bhuva B, Barnes JJ, Patel S. The use of limited cone beam computed tomography in the diagnosis and management of a case of perforating internal root resorption. Int Endod J 2011;44:777-86. 
39. Cymerman JJ, Cymerman DH, O'Dwyer RS. Evaluation of odontogenic maxillary sinusitis using cone-beam computed tomography: three case reports. J Endod 2011;37:1465-9.

40. Pasqualini D, Scotti N, Ambrogio P, Alovisi M, Berutti E. Atypical facial pain related to apical fenestration and overfilling. Int Endod J 2012;45:670-7.

41. Use of cone-beam computed tomography in endodontics Joint Position Statement of the American Association of Endodontists and the American Academy of Oral and Maxillofacial Radiology. Oral Surg Oral Med Oral Pathol Oral Radiol Endod 2011;111:234-7.

42. Bueno MR, Estrela C, De Figueiredo JA, Azevedo BC. Map-reading strategy to diagnose root perforations near metallic intracanal posts by using cone beam computed tomography. J Endodont 2011;37:85-90.

43. Parirokh $M$, Ardjomand $K$, Manochehrifar $H$. Artifacts in Cone-Beam Computed Tomography of a Post and Core Restoration: A Case Report. Iran Endod J 2012;7:98-101.

\section{Yazışma Adresi}

Yrd. Doç. Dr. Elif TARIM

İzmir Katip Çelebi Üniversitesi

Diş Hekimliği Fakültesi

Ağız Diş ve Çene Radyolojisi Anabilim Dalı, İzmir, Türkiye.

Telefon: +90.232 .3254040$

Faks: +90.232 .325 4040- 2352

E-mail: dteliftarim@yahoo.com 